\title{
Deciding whether Graph $G$ Has Page Number One is in NC
}

\author{
増山 繁 (豊橋技術科学大学知識情報工学系) \\ Shigeru MASUYAMA \\ Department of Knowledge-Based Information Engineering \\ Toyohashi University of Technology \\ Toyohashi 441, Japan \\ 内藤 昭三 (NTT 基礎研究所) \\ Shozo NAITO \\ Basic Research Laboratories \\ NTT \\ Musashino 180, Japan
}

\begin{abstract}
Based on a forbidden subgraph characterization of a graph to have one page, we develop a polylog. time algorithm to tell if page number of given graph $G$ is one with polynomial number of processors, clarifying this problem to be in NC.
\end{abstract}

\section{Introduction}

This paper discusses the problem of deciding whether the given graph is noncrossing, where graph $G$ is noncrossing if there exists a linear arrangement of vertices so that no pair of edges is crossing when they are drawn on the same side of the linear arrangement of vertices. Similar problem setting appears in the formulation of the noncrossing constraint on word modification to sentence generation in natural language processing, which motivates 
us to study this problem.

This problem is a specialization of the book embedding[12] in the sense that this problem asks if the given graph has page number one, i.e., a graph can be embedded in a single page. In general, the book embedding is hard: it is NP-complete to tell if a planar graph can be embedded in two pages [3]. It is known[2, 3, 12] that noncrossing graphs on a single page are exactly the outerplanar graphs[6]. However, no polylog parallel decision algorithm to tell if a graph is outerplanar is known.

The problem of deciding whether given graph $G$ has page number one is formally defined as follows:

Given graph $G=(V, E)$, decide whether $G$ has page number one, where $G$ has page number one if there exists a linear arrangement of vertices by which no pair of edges $e, e^{\prime} \in E$ satisfies $s(e)<s\left(e^{\prime}\right)<t(e)<t\left(e^{\prime}\right)$, where $s(e)(t(e)$, respectively, $)$ is the smaller (the larger) end vertex of edge $e$ in the arrangement.

We first illustrate a characterization of a graph to have page number one by two forbidden subgraphs. Based on this characterization we develop a polylog time algorithm with polynomial number of processors, clarifying this problem to be in NC.

Graphs considered in this paper are undirected and may have multiple edges. We also assume that a path denotes a simple path throughout this paper. CREW PRAM (see e.g., [5]) is adopted as a parallel computation model.

\section{Forbidden Sub- graph Characterization of a Graph to have Page Num- ber One}

We first introduce a characterization of outerplanar graphs[6].

Theorem 1.[6] Given graph $G=$ $(V, E), G$ is outerplanar

if and only if

$G$ has no subgraph homeomorphic to either $K_{4}$ or $K_{2,3}$, where $K_{4}$ is the complete graph on four vertices and $K_{2,3}$ is the graph illustrated in Fig. 1.

Corollary 1. Given biconnected graph $G=(V, E), G$ is outerplanar

if and only if 
$G$ has no subgraph homeomorphic to either $K_{4}$ or $K_{2,3}$.

As $G$ has page number one if and only if $G$ is outerplanar[2,3,12] (, we call this Fact 1 ), and $K_{4}$ is a forbidden subgraph of a series parallel graph[4] we have the following corollaries.

Corollary 2. If $G=(V, E, s, t)$ is a series parallel graph $[4,10]$ and has no subgraph homeomorphic to $K_{2,3}$, then $G$ has a one page embedding in which the left terminal $s$ of $G$ has the smallest number.

Corollary 3. Given series parallel graph $G=(V, E), G$ has page number one

if and only if

$G$ has no subgraph homeomorphic to $K_{2,3}$.

We now characterize a graph to have page number one for general graphs.

Combining Theorem 1 to Fact 1, we have the following Theorem.

Theorem 2. Given graph $G=(V, E)$, $G$ is noncrossing if and only if

$G$ has no subgraph homeomorphic to either $K_{4}$ or $K_{2,3}$.

No proof of Theorem 1 is provided in [6] and we supplement a constructive proof of Theorem 2, which ensures that Steps 2, 3 of the algorithm in the next section can be performed for each biconnected component $B_{i}$.

(Proof of Theorem 2) Necessity is obvious. To prove the sufficiency, we first note that the following three observations hold when $G$ has no subgraph homeomorphic to $K_{4}$ or $K_{2,3}$.

Observation 1. Each biconnected component of $G$ has no subgraph homeomorphic to $K_{4}$ and is a series parallel graph.

Observation 2. We can obtain a series parallel graph from each biconnected component of $G$ by choosing any vertex as one of its terminal[7].

Observation 3. As each biconnected component of $G$ has no subgraph homeomorphic to $K_{2,3}$, it has a one page embedding by which any vertex can be arranged first (by Observation 2 and Corollary 1 of Theorem 1).

Based on these observations, we shall prove the sufficiency by induction on 
the number of biconnected components in $G$.

(1) If $G$ has exactly one biconnected component, then the sufficiency holds by Corollary 1 of Theorem 1 .

(2) We assume that the sufficiency holds when the number of biconnected components is $n-1$ and consider the case when the number of biconnected components of $G$ is $n$.

Let $S(G)$ be a graph each of whose vertex corresponds to either a biconnected component of $G$ or an articulate vertex of $G$, where $(B, a)$ is an edge of $S(G)$ if and only if articulate vertex $a$ belongs to biconnected component $B$. Note that $S(G)$ is a tree.

Let $B$ be one of the leaves of $S(G)$ (, note that $B$ must correspond to a biconnected component of $G$,) and let $a$ be an articulate vertex belonging to $B$. Let $G-B$ be a graph obtained by removing $B$, except $a$, from $G$. $G-B$ has $n-1$ biconnected components and has page number one. On the other hand, $B$ has a one page embedding where $a$ is arranged first. Thus by embedding this one page embedding of $B$ in the one page embedding of $G-B$ between $a$ and the vertex next to $a$ in the arrangement, we have shown that $G$ has page number one.

\section{Deciding if a Graph has Page Number One is in NC}

We now introduce a linear time algorithm, by which we can decide, with respect to the number of edges, whether the given graph $G$ has page number one. In the algorithm, $a(G)$ is the number of vertex disjoint paths with length at least two in $G$ connecting its terminals and $b(G)=1$, if $G$ has a subgraph homeomorphic

to $G_{5}$ in Fig. 2. Otherwise, $b(G)=0$.

\section{Algorithm PNO}

Step 1. Decompose $G$ into biconnected components $B_{1}, B_{2}, \ldots, B_{k}$ and construct $S(G)$ defined in the proof of Theorem 2.

for each $B_{i}, i=1, \ldots, k$ let $G \leftarrow B_{i}$ and do Steps 2, 3:

Step 2. Decide whether $G$ is a series parallel graph, and if $G$ is a series parallel graph, then construct the parse tree of $G$ which describes how to construct $G$ from the set of edges of $G[9,10]$.

Step 3. Construct $G$ in accordance with the parse tree in a bottom up manner.

Step 3.1. $a(e) \leftarrow 0, b(e) \leftarrow 0$, for each edge $e$ of $G$.

Step 3.2. When series connection of $G^{\prime}$ and $G^{\prime \prime}$ is performed, let $G$ be the resulting graph.

$$
a(G) \leftarrow 1 .
$$

If either $a\left(G^{\prime}\right)=2$ or $a\left(G^{\prime \prime}\right)=2$ 
holds,

$$
b(G) \leftarrow 1 .
$$

Step 3.3. When parallel connection is performed, let $G$ be the resulting graph obtained from $G^{\prime}$ and $G^{\prime \prime}$. If either $b\left(G^{\prime}\right)=1$ or $b\left(G^{\prime \prime}\right)=1$ holds, then print " $G$ has a page number more than one" and stop.

$$
a(G) \leftarrow a\left(G^{\prime}\right)+a\left(G^{\prime \prime}\right)
$$

If $a(G) \geq 3$, then print " $G$ has page number more than one" and stop.

Step 3.4. If the root of the parse tree is already visiter', then $G$ has a page number one.

The correctness of the algorithm immediately follows Corollary 1 of Theorem 1 and Theorem 2.

To implement the above algorithm in parallel, note that:

Step 1 can be performed in $O\left(\log ^{2} n\right)$ time using $O\left(n^{2} / \log ^{2} n\right)$ processors by [11], where $n$ is the number of vertices in $G$.

"for statement" before Step 2 should be replaced by

for each $B_{i}, i=1, \ldots, k$ in parallel do let $G \leftarrow B_{i}$ and do Steps 2, 3:

Step 2 can be performed $O\left(\log ^{2} n+\right.$ $\log m)$ time with $O(n+m)$ processors, where $n(m)$ is the number of vertices (edges) in $G$. by applying the recognition algorithm of series parallel graphs in [7] (note that although the first part of the algorithm in [7] may be simplified as $G$ is biconnected, the overall complexity is not improved),

Step 3 can be computed in $O(\log l)$ time with $O(l / \log l)$ processors, where $l$ is the number of vertices in the parse tree, by applying tree contraction algorithm in [1] (see also [7].) As $l$ is $O(n)$, this step can be computed in $O(\log n)$ time with $O(n / \log n)$ processors,

In total, this algorithm works in $O\left(\log ^{2} n+\log m\right)$ time with $O\left(n^{2} / \log ^{2} n+m\right)$ processors on CREW PRAM.

\section{Concluding Remarks}

An actual one page embedding of given graph $G$ which consists of more than one blocks can be obtained, if it exists, by appending the following Step 4 to Algorithm PNO.

Step 4. Merge one page embeddings of $B_{i}$ 's, in a manner described in the proof of Theorem 2 , by visiting vertices of $S(G)$ in preorder from some arbitrary articulate vertex $r$.

Step 4 can be performed in parallel by obtaining preorder numbering of vertices of $S(G)$ and constructing lineary ordered list $L$ of vertices of $S(G)$ where vertices are arranged in ascend- 
ing order of its preorder numbering. This can be done by first applying Euler tour technique[11,5] on tree $S(G)$ and doubling technique in a manner described in [5]. This can be done in $O(\log k)$ time by $O(k)$ processors, where $k$ is the number of biconnected components of $G$. As $k \leq n$, this step can be performed in $O(\log n)$ time by $O(n)$ processors.

Then we replace, in parallel, each $B_{i}$ by its one page embedding. To do this, note that each $B_{i}$ can be replaced in the following manner as described in the proof of Theorem 2 :

for each articulate vertex $j$ in parallel do

Make a one page embedding of each $B_{i}$ beginning at $j$ such that $j$ is the father of $B_{i}$ in the rooted tree obtained from $S(G)$ by Euler tour technique [5]. Then substitute, in parallel, each $B_{i}$ with list $L$ of vertices of the one page embedding. Finally, remove the articulate vertex $j$ from the list $L$.

This can be done in constant time. Thus both the overall time complexity and the number of processors required coincide with those of Algorithm PNO.

\section{References}

[1] K. Abramson, N. Dadoun, D. G. Kirkpatrick and T. Przytycka, A simple parallel tree contraction al- gorithm, J. of Algorithms 10, 287302 (1989).

[2] F. Bernhart and P. C. Kainen, The book thickness of a graph, J. Combin. Theory, Ser. B, 27, pp. 320331 (1979).

[3] F. R. K. Chung, F. T. Leighton, and A. L. Rosenberg, Embedding graphs in books: A graph layout problem with applications to VLSI design, SIAM J. Algebraic and Discrete Methods, 1986.

[4] R. J. Duffin, Topology of seriesparallel networks, J. of Mathematical Analysis and Applications 10, 303-318 (1965).

[5] A. Gibbons and W. Rytter, Efficient Parallel Algorithms, Cambridge University Press (1988).

[6] F. Harary, Graph Theory, Addison-Wesley (1959).

[7] X. He, Efficient parallel algorithms for series parallel graphs, $J$. of $\mathrm{Al}$ gorithms 12, 409-430 (1991).

[8] X. He and Y. Yesha, Parallel recognition and decomposition of two terminal series parallel graphs, Information and Computation 75, 15-38 (1987).

[9] J. E. Hopcroft and R. E. Tarjan, Dividing a graph into tri- 
connencted components, SIAM J.

Comput., 2, 135-158 (1973).

[10] T. Kikuno, N. Yoshida and Y. Kakuda, A linear algorithm for the domination number of a seriesparallel graph, Discrete Applied Mathematics 5, 299-311 (1983).

[11] R. E. Tarjan and U. Vishkin, Finding biconnected components and computing tree functions in logarithmic parallel time, SIAM J. Comput. 13, 580-599 (1985).

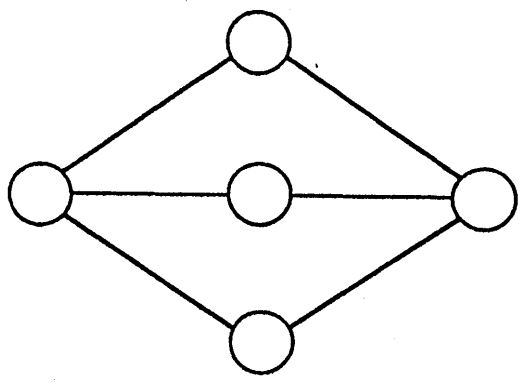

Fig. 1. Forbidden subgraph $K_{2,3}$

[12] M. Yannakakis, Embedding Planar Graphs in Four Pages, J. of Computer and System Sciences 38, 36-67, 1989.

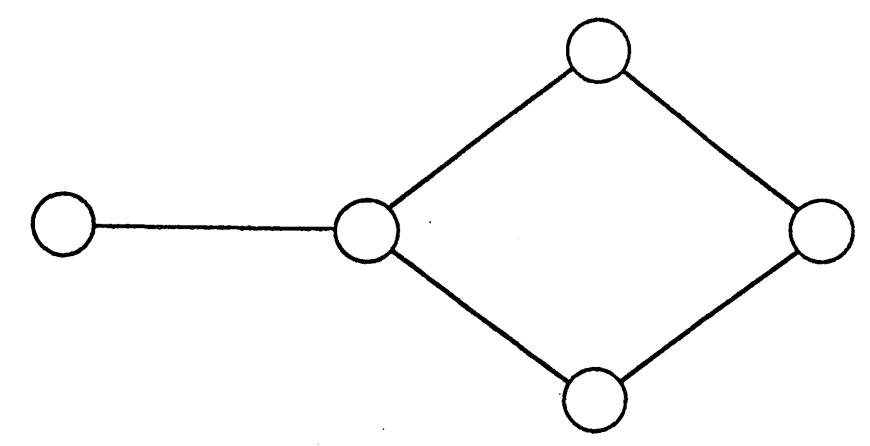

Fig. 2. $\mathrm{G}_{5}$ 